\title{
EMPREENDEDORISMO DE BASE TECNOLÓGICA NO SUL DO BRASIL
}

\author{
Data de submissão: 19/09/2017 \\ Aceite: 17/07/2018 \\ Julio Cezar Mairesse Siluk ${ }^{1}$ \\ Taís Bisognin Garlet ${ }^{2}$ \\ Rafael Marcuzzo ${ }^{3}$ \\ Cláudia de Freitas Michelin ${ }^{4}$ \\ Italo Fernando Minello ${ }^{5}$
}

\section{RESUMO}

Este artigo analisa o panorama das incubadoras de empresas de base tecnológica (IBTs) na região sul do Brasil sob um viés socioeconômico e intelectual, através de correlações com instituições de ensino superior com programas de pós-graduação stricto sensu, direitos de propriedade intelectual concedidos, investimento em ciência e tecnologia, Produto Interno Bruto (PIB) e Índice de Desenvolvimento Humano (IDH). O estudo focou na região sul-brasileira, visto que esta apresenta alguns dos melhores indicadores sociais do país. Através da análise de correlações, pode-se elucidar um cenário de destaque nas nações desenvolvidas, que envolve o empreendedorismo de base tecnológica. Os resultados demonstraram que: (1) a presença de IBTs está fortemente relacionada à existência de instituições de ensino superior com programas de pós-graduação stricto sensu; (2) a existência de IBTs está altamente relacionada a níveis elevados de produção intelectual; (3) estados que recebem mais investimentos em ciência e tecnologia estão positivamente relacionados com IBTs; (4) a existência de IBTs apresenta correlação desprezível com o PIB; (5) a existência de IBTs apresenta correlação fraca com o IDH. Assim, o estudo permitiu verificar o cenário sul-brasileiro com relação à implantação e ao funcionamento de IBTs.

Palavras-chave: Incubadoras de empresas de base tecnológica; indicadores socioeconômicos; propriedade intelectual; investimento em ciência e tecnologia.

\footnotetext{
1 Possui graduação em Administração pela Universidade Federal de Santa Maria, UFSM, mestrado em Engenharia de Produção pela UFSM e doutorado em Engenharia de Produção pela Universidade Federal de Santa Catarina, UFSC. Santa Maria - Rio Grande do Sul. Brasil. E-mail: jsiluk@ufsm.br

2 Possui graduação em Engenharia Química pela Universidade Federal de Santa Maria, UFSM, mestrado em Engenharia de Produção pela UFSM e doutorado em andamento em Engenharia de Produção na Universidade Federal do Rio Grande do Sul, UFRGS. Porto Alegre - Rio Grande do Sul. Brasil. E-mail: tais_garlet@hotmail.com

3 Possui graduação em Engenharia de Produção pela Universidade Federal de Santa Maria, UFSM e mestrado em Engenharia de Produção pela UFSM. Santa Maria - Rio Grande do Sul. Brasil. E-mail: rafael.marcuzzo@mail.ufsm.br

4 Possui graduação em Ciências Contábeis pela Unidades Integradas de Ensino Superior do Vale do Jacuí, UNIVALE, Mestrado em Engenharia de Produção pela Universidade Federal de Santa Maria e Doutorado em Administração pela Universidade Federal de Santa Maria. Cachoeira do Sul - Rio Grande do Sul. Brasil E-mail: claudiamichelin@ulbra.edu.br

5 Possui graduação em Administração de Empresas pela Universidade Federal de Santa Maria, UFSM, mestrado em Administração pela Universidade Federal de Santa Catarina, UFSC e doutorado em Administração pela Faculdade de Economia, Administração e Contabilidade da Universidade de São Paulo, FEA-USP. Santa Maria - Rio Grande do Sul. Brasil. E-mail: italo.minello@uol.com.br
} 


\section{INTRODUÇÃO}

No atual mercado globalizado, o interesse na criação de novos negócios está mais intenso do que em qualquer outro momento nos últimos 30 anos (MAS-VERDÚ; RIBEIRO-SORIANO; ROIG-TIERNO, 2015). As organizações buscam, cada vez mais, um diferencial para produzirem e comercializarem seus produtos e serviços, visando maior lucro (MARCUZZO; DOS SANTOS; SILUK, 2017).

As empresas de base tecnológica (EBTs) são exemplos dessa intensificação de novos negócios. Esses empreendimentos visam parcerias com instituições de ensino e pesquisa e com organismos de apoio, como as incubadoras de empresas de base tecnológica (IBTs), para minimizar os diversos riscos aos quais estão suscetíveis. As incubadoras de empresas visam, por sua vez, facilitar a criação e a disseminação de novos empreendimentos (STAL; ANDREASSI; FUIINO, 2016).

Para obter eficácia em termos de investimentos nessas iniciativas, é preciso que haja uma clara definição inicial dos objetivos para diversificação dos fundos de apoio público, que reconheçam as diversas condições econômicas das regiões em que são implementadas (MUNARI; TOSCHI, 2015). Além disso, os governos devem reconhecer o fato de que as empresas, especialmente menores e mais jovens, precisam de subsídios para pesquisa, desenvolvimento e inovação (PD\&I) para progredirem (CZARNITZKI; DELANOTE, 2015).

Sendo assim, as universidades e os centros de pesquisa têm possibilitado o desenvolvimento de tecnologias similares às de grandes empresas, promovendo experiências e conhecimento de soluções técnicas, organizacionais e de mercado (LÖÖF; NABAVI, 2014).Diante disso, um tema central na análise de economias emergentes tem sido a importância de cultivar capacidades em ciência, tecnologia e inovação (PONOMARIOV; TOIVANEN, 2014), uma vez que essa política tem papel importante na prosperidade de uma nação no mercado global (SALAMI; SOLTANZADEH, 2012).

Nesse sentido, o objetivo deste artigo é analisar o panorama das incubadoras de empresas de base tecnológica na região sul do Brasil sob um viés socioeconômico e intelectual. A análise se dá através da realização de correlações entre IBTs e os seguintes indicadores: instituições de ensino superior com programas de pós-graduação stricto sensu, quantidade de direitos de propriedade intelectual concedidos, investimento em ciência e tecnologia, Produto Interno Bruto (PIB) e Índice de Desenvolvimento Humano (IDH). O estudo tem como foco a região sul do Brasil, que abrange os estados do Rio Grande do Sul, de Santa Catarina e do Paraná, uma vez que essa região apresenta os melhores indicadores sociais do Brasil, como, por exemplo, as menores taxas de mortalidade infantil e analfabetismo, os melhores indicadores de saúde, a segunda maior renda per capita e altos Índices de Desenvolvimento Humano. Além disso, é a região responsável por 16,6\% do Produto Interno Bruto nacional (BRASIL, 2015a). Dessa forma, a região sul apresenta condições favoráveis para a análise de fatores impactantes na implantação de instituições que agregam conhecimento, capital intelectual e inovação tecnológica.

Neste artigo, a seção dois introduz uma revisão a respeito de incubadoras de base tecnológica, instituições de ensino superior com programas de pós-graduação stricto sensu, propriedade intelectual, investimento em ciência e tecnologia e indicadores socioeconômicos, bem como desenvolve as hipóteses deste estudo. A seção três detalha a metodologia da pesquisa, descrevendo cada uma das etapas utilizadas para atingir o objetivo do trabalho. A seção quatro apresenta os resultados e as discussões, enquanto que a seção cinco expõe as considerações finais, destacando as implicações do presente estudo. 


\section{REVISÃO BIBLIOGRÁFICA E DESENVOLVIMENTO DAS HIPÓTESES}

Este artigo revisa cinco aspectos importantes para o crescimento local, regional e nacional no âmbito da pesquisa e desenvolvimento de novas tecnologias: empreendedorismo de base tecnológica, instituições de ensino superior, propriedade intelectual, desenvolvimento da ciência e tecnologia e indicadores socioeconômicos. Dentro das temáticas selecionadas para o estudo, estão contidas as hipóteses desenvolvidas para a análise de correlações.

\subsection{Empreendedorismo de base tecnológica}

O empreendedorismo de base tecnológica recebe grande atenção acadêmica por estar no centro do desenvolvimento econômico liderado pela inovação (SMITH et al., 2013; STOKAN; THOMPSON; MAHU, 2015). Nessa perspectiva, iniciativas para incubação devem estar no cerne das políticas de tecnologia e inovação para promover o empreendedorismo, apoiar o desenvolvimento de novas IBTs, fortalecer as ligações entre academia e indústria e estimular atividades de inovação (SCHWARTZ, 2013).

As incubadoras de empresas são vistas como organizações que constituem ou criam um ambiente propício para a incubação e o desenvolvimento de novas empresas (BERGEK; NORRMAN, 2008). Nesse sentido, devem oferecer diversos serviços de suporte técnico destinados a expandir as novas e emergentes empresas em condições financeiras e operacionais independentes (MASUTHA; ROGERSON, 2015). A presença dessas empresas é extremamente importante para uma região e necessita ser apoiada, de maneira a contribuir para o desenvolvimento de novas tecnologias e produtos, criação de empregos e benefícios para as comunidades (BERGEK; NORRMAN, 2008; DELIGIANNI; VOUDOURIS; LIOUKAS, 2015).

As EBTs trabalham com a aplicação intensiva de conhecimento científico e técnico para produzir bens e serviços que, consequentemente, têm vantagem sobre os produtos existentes no mercado (UBEDA et al., 2013). Colovic e Lamotte (2015) complementam que uma grande quantidade de conhecimento é produzida pelas empresas incubadas, entretanto esse conhecimento nem sempre transborda para outros potenciais empreendedores, pois é utilizado para fins comerciais, primordialmente. Todas as transições eficazes do laboratório para o mercado são importantes para o crescimento econômico do país (WOUTERS; KIRCHBERGER, 2015).

Em termos de atuação internacional, uma característica das EBTs de países com economias emergentes é o fato de suas estratégias tecnológicas e de mercado serem, na maioria dos casos, pautadas pela imitação. Isso leva a dificuldades para tais empresas criarem seus mercados, visto que esbarram em concorrentes externos mais estruturados e de maior porte que fornecem, nos mercados estrangeiros, o mesmo produto ou um similar (CAHEN; LAHIRI; BORINI, 2016).

No caso das EBTs brasileiras, as oportunidades dão-se principalmente em mercados nos quais a demanda nacional é pequena demais para justificar a internalização da operação dos concorrentes estrangeiros. Ou, ainda, quando há mercados nos países desenvolvidos que não apresentam interesse suficiente para atuação de empresas mais estruturadas, sendo nichos também potenciais de sucesso para as EBTs brasileiras (RIBEIRO; OLIVEIRA JR.; BORINI, 2012). 


\subsection{Instituições de ensino superior}

As universidades são uma fonte de conhecimento científico de extrema importância e estão fortemente ligadas a institutos de pesquisa públicos, proporcionando conhecimento e recursos a negócios inovadores, como as empresas de base tecnológica (LÖFSTEN, 2016). Tais instituições de ensino servem de baliza para a concepção e implementação de políticas de ciência e tecnologia adequadas, através do fornecimento de informações sobre o contexto para colaboração com a indústria (FREITAS; MARQUES; SILVA, 2013).

Além disso, a excelência científica é um fator crítico para a descoberta de oportunidades tecnológicas para a indústria (D'ESTE et al., 2012), tendo maiores níveis de atividade em programas de pós-graduação. Desse modo, instituições que ofertam cursos de pós-graduação, em especial na modalidade stricto sensu, possuem relação direta com pesquisa e desenvolvimento e podem prover novas tecnologias para as empresas e melhorias à sociedade.

No Brasil, de acordo com o Sistema de Informações Georreferenciadas (2015), existem 3.302 instituições de ensino superior com programas de pós-graduação stricto sensu, ou seja, aqueles que incluem apenas cursos de mestrado e doutorado acadêmicos. Dessas, 708 estão localizadas nos estados da região sul do país, equivalendo a aproximadamente $21,5 \%$ das instituições brasileiras com esse perfil.

A presença da universidade também é importante no que se refere à qualificação da força de trabalho, ao efeito gerado por suas pesquisas para o aumento da produtividade, à solução de problemas locais, ao aumento do nível geral de conhecimento e de cultura da região e a sua contribuição enquanto lócus de inovação (ROLIM; SERRA, 2015). Essas informações induzem a crença de que há uma relação positiva entre a existência de IBTs e a presença de instituições de ensino superior com cursos de pós-graduação stricto sensu nos municípios pertencentes aos estados da região sul do Brasil. Assim, criam-se as seguintes hipóteses:

Hipótese 1(a): A existência de incubadoras de empresas de base tecnológica está positivamente relacionada à existência de instituições de ensino superior com programas de pós-graduação stricto sensu.

Hipótese 1(b): As cidades com maior quantidade de instituições de ensino superior com programas de pós-graduação stricto sensu possuem incubadoras de empresas de base tecnológica.

\subsection{Propriedade intelectual}

A Propriedade Intelectual (PI) compreende um conjunto de direitos legalmente reconhecidos quando ideias ou invenções são protegidas (OH; MATSUOKA, 2016). É vista como uma ferramenta poderosa para o crescimento econômico e possibilita a obtenção de recursos relevantes para as empresas através da interação dinâmica entre pesquisa de excelência, atividade empresarial e estratégias públicas de apoio. Assim, a excelência em pesquisa proporciona avanços tecnológicos extremamente importantes, contribuindo para a criação de novos mercados e novas oportunidades de trabalho (D'ESTE et al., 2012).

No Brasil, de acordo com o Instituto Nacional da Propriedade Intelectual (BRASIL, 2015b), no período compreendido entre 2000 e 2012, foram concedidos 505.206 direitos de propriedade intelectual, englobando patentes de invenção, modelos de utilidade, certificados de adição, marcas, desenhos industriais, certificados de tecnologia e programas de computador. Os direitos de propriedade intelectual são reconhecidos por induzir a divulgação de novas tecnologias, estimular a difusão do conhecimento e elevar a estratégia concorrencial das empresas (BRÜGGEMANN et al., 2016; KAY; YOUTIE; SHAPIRA, 2014). 
Na região sul do Brasil, foco deste estudo, tem-se $21,82 \%$ das concessões, estando elas distribuídas entre os seus três estados da seguinte forma: 7,65\% no Rio Grande do Sul, 6,22\% em Santa Catarina e 7,95\% no Paraná. Para compararmos tal nível de atividade, a média nacional de direitos de propriedade intelectual concedidos no mesmo intervalo de tempo é de 1.439 , enquanto que a média apresentada pela região sul do país é de 2.828. Isso indica que os estados da região sul tiveram nível de atividade em PI aproximadamente $97 \%$ maior que os demais estados brasileiros (BRASIL, 2015b).

É importante salientar que a PI está fortemente protegida pelas universidades, que estão cada vez mais comprometidas em promover novas iniciativas com vistas ao desenvolvimento econômico regional e nacional (HUANG; CHENG, 2015). Diante disso, infere-se que há uma relação positiva entre a existência de IBTs e a produção de PI nos municípios da região sul do Brasil. Dessa forma, estipulam-se as seguintes hipóteses:

Hipótese 2(a): A existência de incubadoras de empresas de base tecnológica está positivamente relacionada a níveis elevados de produção intelectual.

Hipótese 2(b): As cidades com melhores índices de propriedade intelectual possuem incubadoras de empresas de base tecnológica.

\subsection{Desenvolvimento da ciência e da tecnologia}

Os governos desempenham um papel central nos sistemas de inovação através de duas atividades principais. Primeiro, eles geram e disseminam novos conhecimentos através de centros públicos de pesquisa, universidades e empresas. Em segundo lugar, eles criam e modificam instituições (como leis, regulamentos e políticas) que apoiam as atividades científicas, incluindo o financiamento (PADILLA-PÉREZ; GAUDIN, 2014). Nesse contexto, a tecnologia desponta como importante ativo nas empresas, dando suporte ao seu crescimento e à retenção de vantagens competitivas, sempre atrelada ao desenvolvimento socioeconômico (WANG; WANG; WU, 2015).

Não obstante, de acordo com Lasrado et al. (2016), atualmente as incubadoras têm um efeito tão significativo no crescimento de emprego que a oferta de vagas chega a ser estatisticamente maior do que a proporcionada pelo setor industrial. Além disso, as agências de financiamento federais, estaduais e locais em todo o mundo estão muito entusiasmadas nesse mercado que atua principalmente através de incubadoras de empresas, parques científicos ou centros tecnológicos (SCHWARTZ, 2013).

No Brasil, o Ministério da Ciência, Tecnologia e Inovação (MCTI), com a incorporação de duas das mais importantes agências de fomento do país - a Financiadora de Estudos e Projetos (FINEP) e o Conselho Nacional de Desenvolvimento Científico e Tecnológico (CNPq) - passou a coordenar o trabalho de execução dos programas e ações que consolidam a Política Nacional de Ciência, Tecnologia e Inovação (PONOMARIOV; TOIVANEN, 2014). Além disso, o governo tem incentivado a cooperação entre empresas e instituições de pesquisa através da Empresa Brasileira de Pesquisa e Inovação Industrial (EMBRAPII, 2015a), que foca na demanda empresarial utilizando a infraestrutura das instituições de pesquisa. Nessa perspectiva, visualizam-se mecanismos nacionais elaborados para propiciar o fomento a novas ideias e desenvolvê-las.

A média de investimentos provenientes das agências de fomento FINEP, CNPq e EMBRAPII, durante o período de 2000 a 2015, totaliza R\$1.454.261.945,67 em todo o Brasil, ao passo que na região sul esse valor é de R\$ 292.851.600,00 (BRASIL, 2016; EMBRAPII, 2015b). Esses dados indicam que os estados da região sul recebem dessas agências de fomento investimento em ciência e tecnologia correspondente a $20,14 \%$ do montante investido no país.

De acordo com o Instituto de Pesquisa Econômica Aplicada (IPEA), boa parte dos investimentos em pesquisa e desenvolvimento ( $P \& D$ ) realizados pelos países é proveniente de universida- 
des e instituições públicas de pesquisa (DE NEGRI; CAVALCANTE; ALVES, 2013). Entretanto, conforme Freitas, Marques e Silva (2013), as unidades empresariais de pesquisa, em forma de centros dedicados ou departamentos, são igualmente essenciais para o desenvolvimento de novos campos científicos.

Diante disso, vislumbra-se uma relação positiva entre os investimentos em ciência e tecnologia e a existência de instituições que visam o desenvolvimento dessas novas ideias que surgem mediante a pesquisa e o desenvolvimento. Assim, para elucidar tal consideração, os autores deste artigo hipotetizam a seguinte relação:

Hipótese 3: Estados que recebem mais investimentos em ciência e tecnologia possuem incubadoras de empresas de base tecnológica.

\subsection{Indicadores socioeconômicos}

Os países necessitam de indicadores que sejam eficientes para mensurar e avaliar seu desenvolvimento sob os pontos de vista econômico, social e ambiental (KUBISZEWSKI et al., 2013). Critérios confiáveis para analisar o desempenho global das nações, das regiões e do planeta como um todo são capazes de proporcionar uma conexão fundamental entre o estabelecimento da economia e a formulação de políticas racionais (GIANNETTI et al., 2015). Assim, indicadores socioeconômicos são formulados para apontar as características básicas do desenvolvimento das sociedades, merecendo destaque pelo uso tradicional o Produto Interno Bruto (PIB) e o Índice de Desenvolvimento Humano (IDH).

O Produto Interno Bruto é o indicador mais utilizado para analisar a evolução da atividade econômica dos países. Além disso, o PIB representa o valor total de mercado de todos os bens e serviços finais produzidos pela economia de um país em um determinado período de tempo, incluindo apenas medidas monetárias (FERREIRA; PEREIRA, 2015). Quando se analisa a proporção do PIB com a população de um país, tem-se o PIB per capita (FRUGOLI et al., 2015).

Já o Índice de Desenvolvimento Humano estabeleceu-se ao longo dos anos como um marcador importante do progresso das sociedades (DERVIS; KLUGMAN, 2011). É composto por três elementos: educação, longevidade e padrão de vida (MANIYALATH; NARENDRAN, 2016). A educação é um recurso relevante para o índice, uma vez que ajuda a proporcionar emprego e conhecimento às pessoas, sendo mensurada pela média dos anos de escolaridade e dos anos esperados. O segundo componente do IDH é a longevidade, estimada pela expectativa de vida dos indivíduos desde seu nascimento. O último elemento é o padrão de vida, calculado em termos de paridade de poder aquisitivo, necessário para ajudar com a compra de bens e serviços fundamentais ao desenvolvimento humano (GAMLATH, 2013; MORSE, 2014).

De acordo com o Instituto Brasileiro de Geografia e Estatística (IBGE), o PIB per capita do Brasil para o ano de 2013 é de $R \$ 26.445,00$, enquanto o valor médio apresentado pela região sul do país é de R\$30.737,25 (BRASIL, 2015a). Esses dados apontam que a região composta pelos estados do Rio Grande do Sul, Santa Catarina e Paraná apresenta indicador de crescimento econômico 16,23\% maior que a média nacional. Ainda segundo o IBGE, no que tange ao IDH, o Brasil, no período de 2010, apresentou um índice de 0,699 , ao passo que a região sul do país exibiu um IDH médio de 0,756 (BRASIL, 2015a). A partir dessas informações, é possível afirmar que essa região é $8,15 \%$ mais desenvolvida, no que diz respeito a esse indicador, que as demais regiões do país. Por meio da observação dos dados de PIB e IDH, nota-se o tamanho do Brasil e as diferenças emergentes das suas características regionais, que englobam traços sociais, econômicos, culturais e significativamente educacionais (SANT'ANNA; RIBEIRO; DUTT-ROSS, 2011).

Indicadores socioeconômicos são, então, de extrema importância para avaliar o desen- 
volvimento dos países e auxiliar na criação de empresas e na geração de oportunidades econômicas. Além disso, atuam como um mecanismo que aponta as regiões mais propícias para receberem investimentos (KLAFKE et al., 2016). Sendo assim, é possível acreditar que há uma relação positiva entre a existência de IBTs e indicadores como o Produto Interno Bruto e o Índice de Desenvolvimento Humano nos municípios da região sul do Brasil. Dessa forma, as seguintes hipóteses foram formuladas:

Hipótese 4: A existência de incubadoras de empresas de base tecnológica está positivamente relacionada a melhores níveis do indicador Produto Interno Bruto.

Hipótese 5: A existência de incubadoras de empresas de base tecnológica está positivamente relacionada a melhores Índices de Desenvolvimento Humano.

\section{METODOLOGIA}

Esta seção descreve as variáveis e o método utilizados na análise, a fim de embasar quantitativamente o estudo. Esta pesquisa empírica classifica-se como correlacional (levantamento), pois sumariza resultados expressando relação entre duas variáveis (ROVINE; EYE, 1997), reconhecendo a linearidade intrínseca a tal método. Além disso, conforme Rovine e Eye (1997), os componentes correlacionados devem ter uma estrutura combinatória conhecida com clareza pelos pesquisadores que deles se utilizam, conforme foi explicado nas seções anteriores. Assim, para o delineamento deste estudo foram realizadas diversas etapas com o propósito de alcance dos resultados, ilustradas na Figura 1.

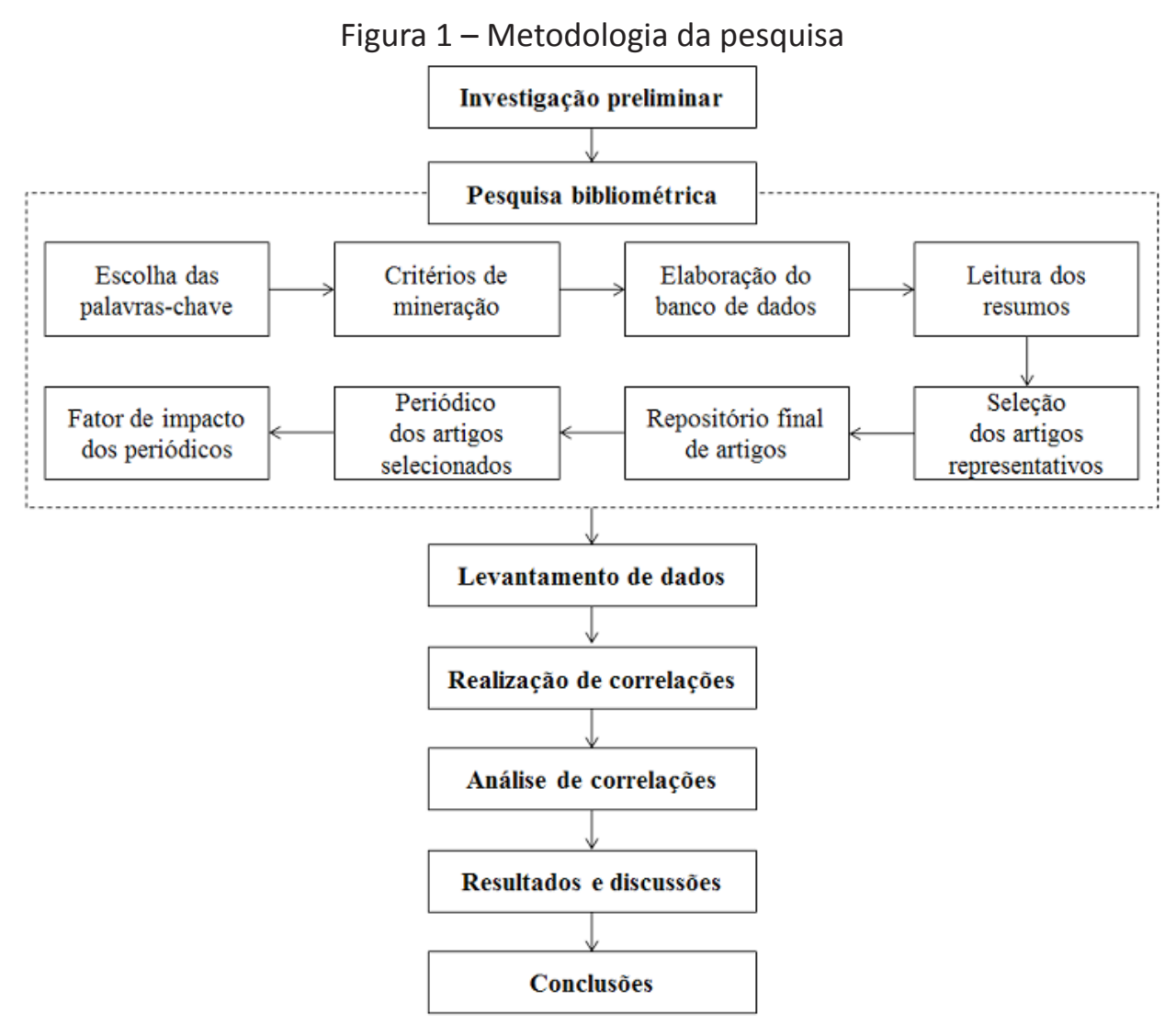

Fonte: Elaborado pelos autores. 


\subsection{Investigação preliminar}

Esta etapa consistiu em uma investigação preliminar acerca do tema, em que foram realizadas buscas em portais de periódicos e em plataformas on-line para verificar publicações atuais a respeito de incubadoras de empresas de base tecnológica associadas a indicadores socioeconômicos e de caráter intelectual. Desse modo, foi possível aproximar as concepções e diretrizes pensadas pelos pesquisadores com o que tem sido publicado por outros autores na área.

\subsection{Pesquisa bibliométrica}

Na segunda etapa da pesquisa fez-se uma revisão bibliométrica, que teve por objetivo justificar e embasar a importância do tema estudado. Foram realizadas as filtragens das informações através da escolha de palavras-chave fundamentadas na temática da pesquisa. Através disso, fez-se uma seleção e uma análise bibliométrica de artigos contemplando os principais mecanismos de busca, como as bases Science Direct e Web of Science, com as palavras-chave technology-based incubators, technology-based firms, socioeconomic indicators, correlation, incubated firms, intellectual property, post-graduation, gross domestic product, human development index, science and technology investments, bem como combinações destes termos. O período compreendido entre 2011 e 2016 foi o delimitador, e se considerou como critérios de seleção os artigos mais relevantes e a correspondência dos termos no título e resumo.

Na sequência, foi elaborado um banco de dados para apresentação dos artigos que se assemelham à pesquisa, e, consequentemente, se realizou a leitura dos resumos para cumprir os objetivos da revisão bibliométrica, gerando um repositório final de 67 artigos. A partir disso, verificou-se a qualidade dos artigos publicados por meio do fator de impacto dos periódicos. Dessa forma, foi analisado o banco de dados com o intuito de identificar abordagens originais e relevantes que se aproximassem da pesquisa proposta.

\subsection{Levantamento de dados}

Esta etapa consistiu em um levantamento atual das IBTs existentes na região sul do Brasil. Para isso, partiu-se da premissa de que tais incubadoras estariam presentes ou sendo apoiadas por instituições de ensino superior. Assim, fez-se uma busca no cadastro e-MEC de instituições e cursos de educação superior para verificar quais instituições de ensino superior estão cadastradas, em quais cidades estavam, e, posteriormente, foram identificadas as incubadoras de base tecnológica vinculadas a essas instituições.

Para as cidades da região sul que contam com a presença de IBTs, foram pesquisados no portal do Instituto Brasileiro de Geografia e Estatística (IBGE) seus indicadores socioeconômicos, como o Produto Interno Bruto e o Índice de Desenvolvimento Humano. Além disso, no sítio eletrônico da Empresa Brasileira de Pesquisa e Inovação Industrial (EMBRAPII) e na Plataforma Aquarius do Ministério da Ciência, Tecnologia e Inovação, fez-se o levantamento do capital investido em ciência e tecnologia pelo Conselho Nacional de Desenvolvimento Científico e Tecnológico (CNPq), pela Financiadora de Estudos e Projetos (FINEP) e pela EMBRAPII no Rio Grande do Sul, Santa Catarina e Paraná, além dos demais estados brasileiros. 
No portal do Instituto Nacional da Propriedade Intelectual (INPI), pesquisou-se, para o período de 2000 a 2012, a quantidade de direitos de propriedade intelectual concedidos em todos os municípios da região sul, bem como para todos os de outros estados do Brasil. Já na Plataforma Sucupira, da Coordenação de Aperfeiçoamento de Pessoal de Ensino Superior (CAPES), fez-se a busca por instituições de ensino superior que apresentam programas de pós-graduação stricto sensu nos municípios da região sul-brasileira e em todos os demais estados do país.

\subsection{Realização de correlações}

A partir dos dados levantados, foram feitas correlações para testar as hipóteses formuladas. Para este passo, foi utilizado o software Statistica, em que, a partir do banco de dados, foram feitos matrizes e gráficos de correlação para analisar a associação entre as variáveis, considerando confiança de $95 \%$. As correlações foram realizadas relacionando o número de IBTs com a quantidade de instituições de ensino superior com programas de pós-graduação stricto sensu, com o índice de direitos de PI concedidos, com o capital investido em ciência e tecnologia pelas agências de fomento FINEP, CAPES e EMBRAPII e com os indicadores PIB e IDH, sendo que todas as variáveis correspondem às cidades que possuem IBTs na região sul do Brasil. Na sequência, para as cidades da região sul que apresentam mais instituições de ensino superior com programas de pós-graduação stricto sensu, assim como para as cidades com maior número de direitos de propriedade intelectual concedidos, fez-se a correlação com a quantidade de IBTs existentes nessas cidades.

\subsection{Análise de correlações}

A análise de correlação refere-se a inferências estatísticas do coeficiente de associação linear, que mede o grau de relacionamento entre duas variáveis. A correlação foi considerada positiva quando os fenômenos variaram no mesmo sentido, ao passo que foi negativa quando os fenômenos variaram em sentido inverso. Dessa forma, a partir dos coeficientes de correlação obtidos na quarta etapa, verificou-se a relação existente entre cada uma das variáveis, de forma a confirmar ou rejeitar as hipóteses formuladas. Após essa fase, foi possível discutir os resultados obtidos e redigir as conclusões do estudo, identificando o cenário das IBTs na região sul do Brasil.

\section{RESULTADOS E DISCUSSÕES}

Esta seção descreve os achados estatísticos e comenta acerca das relações entre as variáveis incluídas na análise, contextualizando os resultados. Demonstrando quantitativamente os dados, a Tabela 1 resume os parâmetros considerados para a discussão. 
Tabela 1 - Parâmetros resultantes das correlações

\begin{tabular}{|c|c|c|c|}
\hline Hipótese & Variável & $\begin{array}{c}\text { Grau de correlação } \\
\text { com IBTs }\end{array}$ & $\begin{array}{c}\text { Intensidade da } \\
\text { correlação }\end{array}$ \\
\hline H1(a) & $\begin{array}{c}\text { Instituições de ensino superior } \\
\text { com programas de pós-graduação } \\
\text { stricto sensu }\end{array}$ & 0,87344 & Forte \\
\hline$H 1(b)$ & $\begin{array}{l}\text { Cidades com mais instituições de } \\
\text { ensino superior com programas de } \\
\text { pós-graduação stricto sensu }\end{array}$ & 0,83160 & Forte \\
\hline $\mathrm{H} 2(\mathrm{a})$ & Propriedade intelectual & 0,76376 & Forte \\
\hline $\mathrm{H} 2$ (b) & $\begin{array}{l}\text { Cidades com mais propriedade } \\
\text { intelectual }\end{array}$ & 0,73025 & Forte \\
\hline H3 & $\begin{array}{c}\text { Investimentos em ciência e } \\
\text { tecnologia }\end{array}$ & 0,97293 & Muito forte \\
\hline H4 & Produto Interno Bruto & 0,08629 & Desprezível \\
\hline H5 & $\begin{array}{c}\text { Índice de Desenvolvimento } \\
\text { Humano }\end{array}$ & 0,39655 & Fraca \\
\hline
\end{tabular}

Fonte: Elaborado pelos autores.

Na primeira hipótese, $\mathrm{H1}(\mathrm{a})$, o grau de correlação encontrado foi de valor 0,87344 , indicando uma relação de forte intensidade entre a existência de incubadoras de empresas de base tecnológica e de instituições de ensino superior com programas de pós-graduação stricto sensu. Isso quer dizer que, nas cidades que possuem IBTs, há um alto grau de assertividade na consideração de que existem instituições de ensino superior com as características selecionadas.

Visto que, ao encontro da afirmação de Fritsch e Aamoucke (2013), as universidades são responsáveis por coletar, gerar e armazenar conhecimento e fazê-lo disponível ao mercado em geral, o resultado da associação nessa correlação indica que as incubadoras de empresas de base tecnológica realmente estão fazendo uso dos recursos de que a universidade dispõe. Entretanto, após décadas de pesquisa, parece que grande parte dos cursos de pós-graduação ainda possui alunos com maiores capacidades de publicação de artigos acadêmicos e de uso de técnicas estatísticas sofisticadas do que de solução de problemas do ambiente de trabalho, diretamente atrelados à atividade empreendedora (COLOVIC; LAMOTTE, 2015). Assim, diante do supracitado e dos resultados encontrados, ratifica-se Mas-Verdú, Ribeiro-Soriano e Roig-Tierno (2015) quando dizem que, se o empreendedorismo baseado no conhecimento constitui um importante veículo para promover o crescimento econômico, uma política exclusiva deve ser formulada e implementada com foco nas start-ups desenvolvidas por acadêmicos em universidades.

Na hipótese $\mathrm{H} 1(\mathrm{~b})$, o estudo também evidenciou uma correlação positiva forte, de valor 0,83160, entre maior nível de atividade acadêmica, representada por programas de pós-graduação stricto sensu, e IBTs. Dessa maneira, é possível afirmar que as cidades que possuem instituições de ensino superior com as características supracitadas estão altamente relacionadas com IBTs.

Esse resultado é coincidente com o estudo de Löfsten (2016), em que os programas de pós-graduação em universidades são compreendidos como uma importante fonte de conhecimento científico, e as EBTs podem utilizar-se desses recursos desenvolvendo conexões com as instituições de ensino superior. Apesar disso, conforme Colovic e Lamotte (2015) afirmam, pouco é conhecido acerca do impacto do nível de desempenho tecnológico de uma nação na criação de EBTs. Nesse sentido, o presente estudo apontou para uma relação clara entre o nível de atividade 
empreendedora acadêmica e o desenvolvimento das áreas tecnológicas, contribuindo para uma possível mudança de paradigma nesse cenário.

Não obstante, Pret, Shaw e Drakopoulou Dodd (2016) e Lasrado et al. (2016) dizem que os pesquisadores de universidades têm muito a ganhar ao estudar os conhecimentos e habilidades necessárias que beneficiam o empreendedorismo em diferentes contextos econômicos. Tal dinamismo pode ser entendido como um ciclo contínuo e retroalimentado, pois, ao passo que a atividade acadêmica melhora o ambiente de empreendedorismo tecnológico, este oferece um imenso campo de trabalho para aquela, principalmente em programas de pós-graduação stricto sensu, conforme este estudo elucidou.

Na hipótese $\mathrm{H} 2(\mathrm{a})$, a análise correlacional entre IBTs e níveis de PI resultou positiva e de grau forte, com valor de 0,76376 . Isto é, nas cidades que possuem IBTs há um nível maior de PI, medido pelos itens considerados no referencial teórico.

Partindo-se dos pressupostos de que os governos de países em desenvolvimento, como o Brasil, são responsáveis pela criação de políticas apropriadas para seu crescimento (SALAMI; SOLTANZADEH, 2012), e de que, de acordo com Padilla-Pérez e Gaudin (2014), o governo é responsável pela criação e modificação das estruturas constituintes de um sistema de inovação, como universidades, há que se dizer que muitas das discussões desses resultados tendem a ser políticas. Entretanto, conforme Sant'anna, Ribeiro e Dutt-Ross (2011), é importante salientar que o Brasil tem uma característica muito variada em relação às suas regiões, devido a sua grande extensão territorial. Assim, com vistas a um julgamento mais correto, considera-se que mais atores tenham mérito, igual ou maior, e até responsabilidades perante a criação, desenvolvimento e ampliação das estruturas e atividades inventivas consideradas no estudo, sendo esses atores, principalmente, empreendedores da iniciativa privada, que muitas vezes colaboram efetivamente para um bom desempenho de projetos provenientes de IBTs em universidades.

Na hipótese H2(b), também foi encontrada uma correlação positiva e de grau forte, com valor de 0,73025 , entre maior nível de PI e IBTs. Em outras palavras, as cidades que possuem maior nível de PI estão altamente relacionadas com IBTs.

A atividade de produção intelectual é um importante aspecto a ser considerado nas estratégias corporativas, pois são fontes de criação e disseminação de conhecimento (KAY; YOUTIE; SHAPIRA, 2014). O estudo em PI compreende uma vasta gama de itens, como programas de computador, patentes, marcas e certificados de tecnologia, provenientes da atividade de pesquisa e desenvolvimento. Diante disso, esse resultado ratifica o proposto por Fritsch e Aamoucke (2013), quando dizem que há uma forte relação entre a presença de atividade de pesquisa e desenvolvimento e os inovadores tipos de negócios emergentes atualmente. Entretanto, é importante considerar, conforme Love e Roper (2015), que os pesquisadores sofrem com altos riscos de incerteza comercial de suas produções, enquanto que as empresas são melhor equipadas para lidar com essa situação. Nesse contexto, de acordo com os resultados encontrados, concorda-se com Kay, Youtie e Shapira(2014) ao salientarem que as empresas devem criar um planejamento estratégico com atenção redobrada para a maximização dos retornos sobre os investimentos em $\mathrm{PI}$, visando reconhecer os investimentos em inovação de processos, produtos e serviços como fatores de posicionamento competitivo.

A hipótese $\mathrm{H} 3$ resultou em uma correlação positiva de grau muito forte, de valor 0,97293, entre IBTs e investimentos em ciência e tecnologia. Ou seja, as cidades que possuem IBTs estão muito altamente relacionadas com maiores investimentos realizados em ciência e tecnologia.

Conforme Pret, Shaw e Drakopoulou Dodd (2016), é necessário considerar as condições e circunstâncias contextuais relevantes de determinado sistema econômico, como geo- 
grafia e aspectos financeiros, para entender o benefício que o capital humano provém a esse sistema. Isso porque, ainda de acordo com os autores, investimentos em ciência propiciam mudanças tecnológicas, que causam alterações de regulação e, por fim, alteram o status quo dos sujeitos e objetos envolvidos no contexto - neste caso, positivamente. A infraestrutura disponível para atividades de P\&D nas instituições de ensino superior, como as IBTs, é propulsora da capacidade de desenvolvimento dos negócios e regiões (VILLANI; RASMUSSEN; GRIMALDI, 2017), refletindo a importância de serem realizados investimentos públicos e privados em ciência e tecnologia.

Por último, as hipóteses $\mathrm{H} 4$ e $\mathrm{H} 5$ apresentaram também uma correlação positiva, porém de graus desprezível $(0,08629)$ e fraco $(0,39655)$, para com os indicadores socioeconômicos PIB e $I D H$, respectivamente. Isso significa dizer que as cidades que possuem IBTs estão pouco ou muito pouco relacionadas com melhores índices dos indicadores socioeconômicos IDH e PIB.

As considerações ao item IDH e sua relação com EBTs são delicadas de serem afirmadas, em detrimento da fragilidade de sua própria composição, conforme alguns autores sugerem (PARUOLO; SAISANA; SALTELLI, 2013; SANT'ANNA; RIBEIRO; DUTT-ROSS, 2011), e expressam pouca relação direta, uma vez que esse índice leva em consideração fatores que são indiretamente impactados ou demoram muito para ser impactados pela atividade de incubação de empresas, como melhoria da qualidade de vida, o que explicaria o resultado. Por outro lado, no indicador PIB e sua relação com EBTs, tem-se uma comparação de certa forma injusta, pois o indicador em questão considera bens produzidos, não realizando mensuração da atividade de pesquisa e desenvolvimento, primordiais nas incubadoras de empresas, que também geram lucro. Mesmo aquelas EBTs que se emanciparam de incubadoras e despontaram para o mercado de trabalho são muitas vezes difíceis de serem identificadas, especificamente em relação a suas origens e ao nível de contribuição para tal índice.

A Figura 2 sumariza as cinco hipóteses da pesquisa, indicando a existência de correlação e suas intensidades, em uma legenda que obedece à seguinte escala: linha pontilhada indica correlação fraca ou desprezível, linha tracejada significa correlação forte e linha contínua representa correlação muito forte.

Figura 2 - Sumário das hipóteses

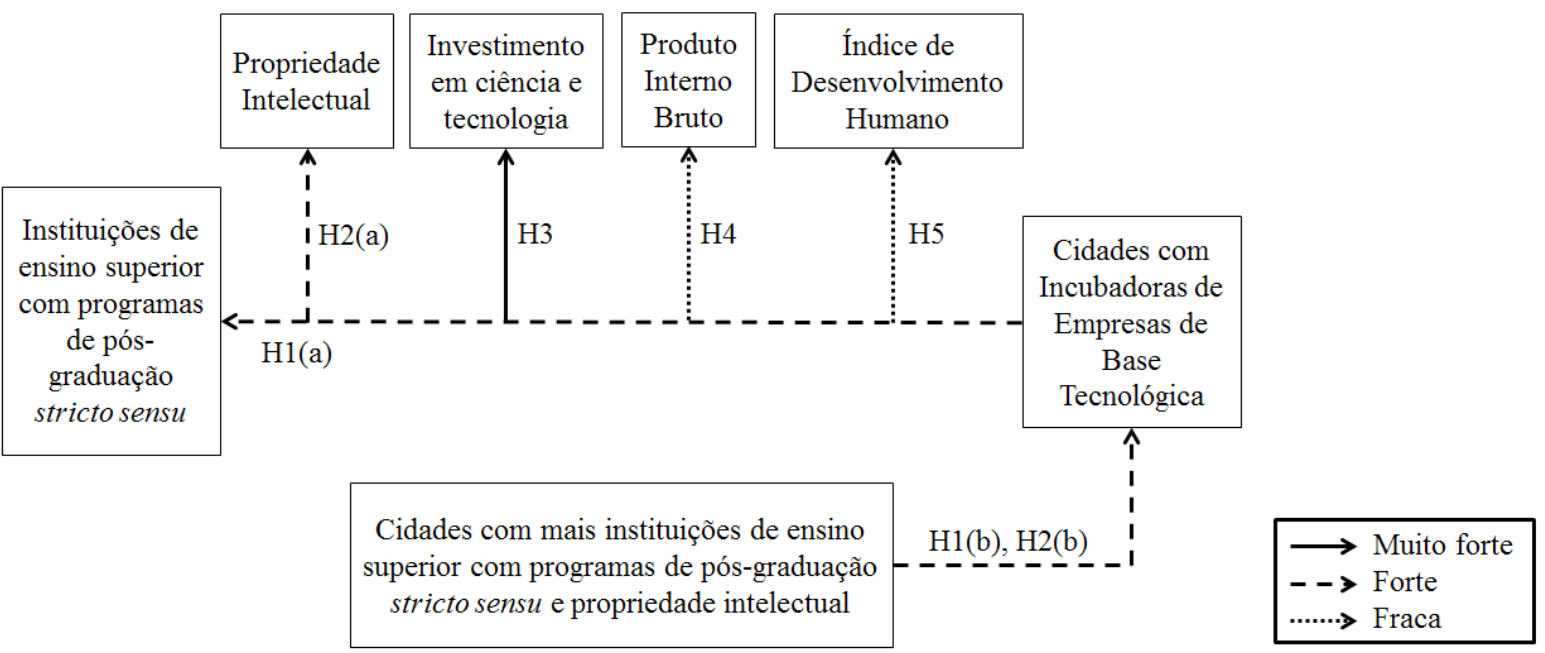

Fonte: Elaborado pelos autores. 
Em suma, os resultados encontrados neste trabalho apontam para uma tendência já existente, porém de relevância, em desenvolvimento no âmbito brasileiro, em comparação a nações mais desenvolvidas, como os Estados Unidos, China e alguns países europeus. Trata-se de uma necessidade de ampliação do entendimento dos atores deste contexto sobre os mecanismos de funcionamento das relações universitárias, empresariais, governamentais e sociais dos países que despontam em melhores níveis. De igual importância, salienta-se que o devido reconhecimento por parte de autoridades públicas sobre como não atrapalhar nessas relações inventivas e produtivas, mas sim estudá-las e estruturar opções de apoio ao desenvolvimento econômico desses setores, também é necessário.

\section{CONCLUSÕES}

O presente estudo objetivou analisar o panorama das incubadoras de empresas de base tecnológica na região sul do Brasil sob um viés socioeconômico e intelectual. Para isso, foram realizadas correlações entre IBTs e os seguintes indicadores: instituições de ensino superior com programas de pós-graduação stricto sensu, quantidade de direitos de propriedade intelectual concedidos, investimento em ciência e tecnologia, Produto Interno Bruto e Índice de Desenvolvimento Humano. O foco da pesquisa foi a região sul do Brasil, que abrange os estados do Rio Grande do Sul, Santa Catarina e Paraná, uma vez que apresenta os melhores indicadores sociais do país. Através da análise de correlações, elucidou-se um cenário que está cada dia mais saliente nas nações desenvolvidas: o empreendedorismo de base tecnológica.

Os aspectos nível de atividade acadêmica em cursos de pós-graduação stricto sensu e nível de produção intelectual apresentaram fortes graus de correlação com as incubadoras de empresas de base tecnológica e implicam considerações relacionadas ao capital humano e ao viés comercial. A ideia de comercialização está associada à captação de recursos adicionais, lacuna recorrente nesses empreendimentos, porém, para tal, existem as agências de transferência de tecnologia, com o objetivo de proporcionar o desenvolvimento econômico local e nacional. Nesse contexto, as universidades devem facilitar a implementação e funcionamento de agências de transferência de conhecimentos e tecnologias através de acordos bilaterais com as incubadoras de empresas.

Enquanto o capital humano impacta a atividade empreendedora, a habilidade para adquiri-lo reflete as turbulências do ambiente em que se encontra determinado ecossistema econômico-social. As considerações nesse aspecto também complementam Love e Roper (2015), no tocante à afirmação de que alguns tipos de conexões com centros de pesquisas em universidades geralmente requerem um bom nível de expertise e capacidade de absorção por parte das novas e pequenas empresas, aumentando os riscos de incertezas ou de insucesso do empreendimento. Tal assertiva indica que as negociações recaem sobre um nível elevado de capacidades, experiências e intuições humanas, indicando que, se estão sendo realizadas, é porque há um bom nível de capital humano e tecnológico sendo desenvolvido na região.

Em relação ao grau de correlação muito forte encontrado entre os investimentos em ciência e tecnologia e as IBTs, compactua-se com Padilla-Pérez e Gaudin (2014) ao afirmarem que o governo dispõe de amplos instrumentos para fortalecer os sistemas de inovação, sejam eles: investimentos públicos, suporte para pequenos e médios negócios, ensino, treinamentos e políticas comerciais e de desenvolvimento regional. Além disso, nos últimos anos, o Brasil tem despontado com uma crescente importância em inovação e produção no comércio internacional (GOEDHUYS; VEUGELERS, 2012). Por fim, afirma-se que as economias emergentes, como a do 
Brasil, devem ser analisadas principalmente a partir de suas capacidades internas de desenvolvimento e cultivo de ciência, tecnologia e inovação.

\subsection{Limitações e futuras pesquisas}

A abrangência geográfica deste estudo foi delimitada como sendo a região sul do Brasil por ser a de mais fácil acesso aos pesquisadores, além de atender aos critérios econômicos e sociais previamente considerados. Diante disso, estas conclusões não podem ser generalizadas em nível de maior abrangência geográfica, como para outras regiões brasileiras ou até mesmo para o país inteiro, uma vez que cada região apresenta características distintas em relação aos itens analisados.

Tendo em vista que esta pesquisa não esgota o tema, nem se constitui em um fim absoluto, para próximos estudos será necessária a investigação in loco das amostras selecionadas através da aplicação de instrumentos como entrevistas semiestruturadas e questionários. Assim, há a oportunidade de verificar se o contexto macro analisado realmente reflete, ou é refletido, pelo ambiente interno das IBTs e seus empreendimentos apoiados.

\section{REFERÊNCIAS}

BERGEK, A.; NORRMAN, C. Incubator best practice: a framework. Technovation, v. 28, p. 20-28, 2008. DOI: 10.1016/j.technovation.2007.07.008.

BRASIL. Instituto Brasileiro de Geografia e Estatística - IBGE. Estados@. Brasília, DF: IBGE, 2015a. Disponível em: <http://www.ibge.gov.br/estadosat/>. Acesso em: 26 set. 2016.

BRASIL. Instituto Nacional da Propriedade Intelectual - INPI. Anuário Estatístico de Propriedade Industrial: 2000-2012. Brasília, DF: INPI, 2015b. Disponível em: <http://www.inpi.gov.br/estatisticas/anuario-estatistico-de-propriedade-industrial-2000-2012-pc2\#pc>. Acesso em: 21 set. 2016.

BRASIL. Ministério da Ciência, Tecnologia e Inovação - MCTI. Plataforma Aquarius. Brasília, DF: MCTI, 2016. Disponível em: <http://aquarius.mcti.gov.br/app/home/>. Acesso em: 4 out. 2016.

BRÜGGEMANN, J. et al. Intellectual property rights hinder sequential innovation. Experimental evidence. Research Policy, v. 45, n. 10, p. 2054-2068, 2016. DOI: 10.1016/j.respol.2016.07.008.

CAHEN, F. R.; LAHIRI, S.; BORINI, F. M. Managerial perceptions of barriers to internationalization: examination of Brazil's new technology-based firms. Journal of Business Research, v. 69, n. 6, p. 1973-1979, June 2016. DOI: 10.1016/j.jbusres.2015.10.143.

COLOVIC, A.; LAMOTTE, O. Technological environment and technology entrepreneurship: a cross-country analysis. Creativity and Innovation Management, v. 24, n. 4, p. 617-628, Dec. 2015. DOI: 10.1111/caim.12133.

CZARNITZKI, D.; DELANOTE, J. R\&D policies for young SMEs: input and output effects. Small Business Economics, v. 45, n. 3, p. 465-485, 2015. DOI: 10.1007/s11187-015-9661-1. 
DELIGIANNI, J.; VOUDOURIS, I.; LIOUKAS, S. Growth paths of small technology firms: The effects of different knowledge types over time. Journal of World Business, v. 50, n. 3, p. 491-504, July 2015. DOI: 10.1016/j.jwb.2014.08.006.

DE NEGRI, F.; CAVALCANTE, L. R.; ALVES, P. F. Relações universidade-empresa no Brasil: o papel da infraestrutura pública de pesquisa. Brasília, Rio de Janeiro: IPEA, 2013. (Textos para discussão, 1901).

D'ESTE, P. et al. Inventors and entrepreneurs in academia: What types of skills and experience matter? Technovation, v. 32, n. 5, May 2012. DOI: 10.1016/j.technovation.2011.12.005.

DERVIS, K.; KLUGMAN, J. Measuring human progress: the contribution of the Human Development Index and related indices. Revue d'économie politique, v. 121, n. 1, p. 73-92, 2011. DOI: 10.3917/redp.211.0073.

EMPRESA BRASILEIRA DE PESQUISA E INOVAÇÃO INDUSTRIAL - EMBRAPII. Plano Diretor 20142019. Brasília, DF: EMBRAPII, 2015a. Disponível em: <http://embrapii.org.br/categoria/institucional/quem-somos/>. Acesso em: 4 out. 2016.

Balanço Patrimonial Ano Referência 2015. Brasília, DF: EMBRAPII, 2015b. Disponível em: <http://embrapii.org.br/categoria/institucional/acesso-a-informacao/>. Acesso em: 4 out. 2016.

FERREIRA, H. M.; PEREIRA, R. S. Paradoxos da dimensão social da sustentabilidade: um estudo longitudinal dos indicadores de desenvolvimento humano no Brasil. Journal on Innovation and Sustainability, v. 6, n. 3, p. 29-49, 2015.

FREITAS, I. M. B.; MARQUES, R. A.; SILVA, E. M. P. University-industry collaboration and innovation in emergent and mature industries in new industrialized countries. Research Policy, v. 42, n. 2, p. 443-453, Mar. 2013. DOI: 10.1016/j.respol.2012.06.006.

FRITSCH, M.; AAMOUCKE, R. Regional public research, higher education, and innovative startups: an empirical investigation. Small Business Economics, v. 41, n. 4, p. 865-885, Dec. 2013. DOI: 10.1007/s11187-013-9510-z.

FRUGOLI, P. A. et al. Can measures of well-being and progress help societies to achieve sustainable development? Journal of Cleaner Production, v. 90, p. 370-380, Mar. 2015. DOI: 10.1016/j. jclepro.2014.11.076.

GAMLATH, S. The governance dimension of human development. Humanomics, v. 29, n. 4, p. 240-259, 2013. DOI: 10.1108/H-03-2013-0015.

GIANNETTI, B. F.; AGOSTINHO, F.; ALMEIDA, C. M. V. B.; HUISINGH, D. A review of limitations of GDP and alternative indices to monitor human wellbeing and to manage eco-system functionality. Journal of Cleaner Production, v. 87, p. 11-25, Jan. 2015. DOI: 10.1016/j.jclepro.2014.10.051. 
GOEDHUYS, M.; VEUGELERS, R. Innovation strategies, process and product innovations and growth: firm-level evidence from Brazil. Structural Change and Economic Dynamics, v. 23, n. 4, p. 516-529, Dec. 2012. DOI: 10.1016/j.strueco.2011.01.004.

HUANG, K.; CHENG, T. Determinants of firms' patenting or not patenting behaviors. Journal of Engineering and Technology Management, v. 36, p. 52-77, 2015. DOI:10.1016/j.jengtecman.2015.05.003.

KAY, L.; YOUTIE, J.; SHAPIRA, P. Signs of things to come? What patent submissions by small and medium-sized enterprises say about corporate strategies in emerging technologies. Technological Forecasting and Social Change, v. 85, p. 17-25, June 2014. DOI: 10.1016/j.techfore.2013.09.006.

KLAFKE, R. V. et al. Primary knowledge management practices applied in Brazil, Russia, India and China (BRIC) industries from 2001-2010. Journal of Knowledge Management, v. 20, n. 4, p. 812828, 2016. DOI: 10.1108/JKM-12-2015-0522.

KUBISZEWSKI, I. et al. Beyond GDP: Measuring and achieving global genuine progress. Ecological Economics, v. 93, p. 57-68, Sept. 2013. DOI: 10.1016/j.ecolecon.2013.04.019.

LASRADO, V. et al. Do graduated university incubator firms benefit from their relationship with university incubators?The Journal of Technology Transfer, v. 41, n. 2, p. 205-219, Apr. 2016. DOI: 10.1007/s10961-015-9412-0.

LÖFSTEN, H. Business and innovation resources. Management Decision, v. 54, n. 1, p. 88-106, 2016. DOI: 10.1108/MD-04-2015-0139.

LÖÖF, H.; NABAVI, P. Survival, productivity and growth of new ventures across locations. Small Business Economics, v. 43, n. 2, p. 477-491, 2014. DOI:10.1007/s11187-014-9553-9.

LOVE, J. H.; ROPER, S. SME innovation, exporting and growth: a review of existing evidence. International Small Business Journal, v. 33, n. 1, p. 28-48, Feb. 2015. DOI: 10.1177/0266242614550190.

MANIYALATH, N.; NARENDRAN, R. The human development index predicts female entrepreneurship rates. International Journal of Entrepreneurial Behavior \& Research, v. 22, n. 5, p. 745-766, 2016. DOI: 10.1108/IJEBR-11-2015-0258.

MARCUZZO, R.; DOS SANTOS, J. R. G.; SILUK, J. C. M. Delineamento para identificação e gerenciamento de ativos intangíveis em empresas de base tecnológica. Revista Científica On-line Tecnologia, Gestão e Humanismo, v. 8, n. 1, 2017.

MAS-VERDÚ, F.; RIBEIRO-SORIANO, D.; ROIG-TIERNO, N. Firm survival: The role of incubators and business characteristics. Journal of Business Research, v. 68, n. 4, p. 793-796, 2015. DOI:10.1016/j. jbusres.2014.11.030.

MASUTHA, M.; ROGERSON, C. M. Business incubation for small enterprise development: South Africa pathways. Urban Forum, v. 26, p. 223-241, 2015. DOI: 10.1007/s12132-014-9242-4. 
MORSE, S. Stirring the pot. Influence of changes in methodology of the Human Development Index on reporting by the press. Ecological Indicators, v. 45, p. 245-254, Oct. 2014. DOI: 10.1016/j. ecolind.2014.04.023.

MUNARI, F.; TOSCHI, L. Assessing the impact of public venture capital programmes in the United Kingdom: Do regional characteristics matter? Journal of Business Venturing, v. 30, n. 2, p. 205226, 2015. DOI:10.1016/j.jbusvent.2014.07.009.

$\mathrm{OH}, \mathrm{C}$.; MATSUOKA, S. Complementary approaches to discursive contestation on the effects of the IPR regime on technology transfer in the face of climate change. Journal of Cleaner Production, v. 128, p. 168-177, Aug. 2016. DOI: 10.1016/j.jclepro.2015.09.050.

PADILLA-PÉREZ, R.; GAUDIN, Y. Science, technology and innovation policies in small and developing economies: the case of Central America. Research Policy, v. 43, n. 4, p. 749-759, May 2014. DOI: 10.1016/j.respol.2013.10.011.

PARUOLO, P.; SAISANA, M.; SALTELLI, A. Ratings and rankings: voodoo or science? Journal of the Royal Statistical Society: Series A (Statistics in Society), v. 176, p. 609-634. June 2013. DOI: 10.1111/j.1467-985X.2012.01059.x.

PONOMARIOV, B.; TOIVANEN, H. Knowledge flows and bases in emerging economy innovation systems: Brazilian research 2005-2009. Research Policy, v. 43, n. 3, p. 588-596, Apr. 2014. DOI: 10.1016/j.respol.2013.09.002.

PRET, T.; SHAW, E.; DRAKOPOULOU DODD, S. Painting the full picture: The conversion of economic, cultural, social and symbolic capital. International Small Business Journal, v. 34, n. 8, p. 10041027, 2016. DOI:10.1177/0266242615595450.

RIBEIRO, F. F.; OLIVEIRA JR., M. M.; BORINI, F. M. Accelerated internationalization of technology-based firms: the case of Brazilian Born-Globals. Revista de Administração Contemporânea, v. 16, n. 6, p. 866-888. Nov./Dec. 2012. DOI: 10.1590/S1415-65552012000600007.

ROLIM, C.; SERRA, M. Ensino superior e desenvolvimento regional: avaliação do impacto econômico de longo-prazo. Revista Brasileira de Estudos Regionais e Urbanos, v. 3, n. 1, 2015.

ROVINE, M. J.; EYE, A. A $14^{\text {th }}$ way to look at a correlation coefficient: correlation as the proportion of matches. The American Statistician, v. 51, n. 1, p. 42-46, Feb. 1997. DOI: 10.2307/2684692.

SALAMI, R.; SOLTANZADEH, J. Comparative analysis for Science, Technology and Innovation policy; Lessons learned from some selected countries (Brazil, India, China, South Korea and South Africa) for other LdCs like Iran. Journal of Technology Management \& Innovation, v. 7, n. 1, p. 211-227, Mar. 2012. DOI: 10.4067/S0718-27242012000100014.

SANT'ANNA, A. P.; RIBEIRO, R. O. A.; DUTT-ROSS, S. Employing the components of the Human Development Index to drive resources to educational policies. Social Indicators Research, v. 104, n. 3, p. 523-532, Dec. 2011. DOI: 10.1007/s11205-010-9759-4. 
SCHWARTZ, M. A control group study of incubators' impact to promote firm survival. The Journal of Technology Transfer, v. 38, n. 3, p. 302-331, June 2013. DOI: 10.1007/s10961-012-9254-y.

SISTEMA DE INFORMAÇÕES GEORREFERENCIADAS. Distribuição de Programas de Pós-graduação no Brasil por Estado. Brasil, 2015. Disponível em: <http://geocapes.capes.gov.br/geocapes2/>. Acesso em: 27 set. 2016.

SMITH, H. L. et al. Entrepreneurial regions: Evidence from Oxfordshire and Cambridgeshire. Social Science Information, v. 52, n. 4, p. 653-673, 2013. DOI: 10.1177/0539018413499978.

STAL, E.; ANDREASSI, T.; FUJINO, A. The role of university incubators in stimulating academic entrepreneurship. RAI Revista de Administração e Inovação, v. 13, n. 2, p. 89-98, 2016. DOI:10.1016/j. rai.2016.01.004.

STATSOFT. Statistica. Disponível em: <http://www.statsoft.com/Products/STATISTICA-Features>. Acesso em: 25 set. 2016.

STOKAN, E.; THOMPSON, L.; MAHU, R. J. Testing the differential effect of business incubators on firm growth. Economic Development Quarterly, v. 29, n. 4, p. 317-327, 2015. DOI: 10.1177/0891242415597065.

UBEDA, J. E. et al. Communication in new technology based-firms. Management Decision, v. 51, n. 3, p. 615-628, 2013. DOI: 10.1108/00251741311309689.

VILLANI, E.; RASMUSSEN, E.; GRIMALDI, R. How intermediary organizations facilitate university-industry technology transfer: A proximity approach. Technological Forecasting and Social Change, v. 114, p. 86-102, 2017. DOI:10.1016/j.techfore.2016.06.004.

WANG, J.; WANG, C.-Y.; WU, C.-Y. A real options framework for R\&D planning in technology-based firms. Journal of Engineering and Technology Management, v. 35, p. 93-114, Jan./Mar. 2015. DOI: 10.1016/j.jengtecman.2014.12.001.

WOUTERS, M.; KIRCHBERGER, M. A. Customer value propositions as interorganizational management accounting to support customer collaboration. Industrial Marketing Management, v. 46, p. 54-67, Apr. 2015. DOI: 10.1016/j.indmarman.2015.01.005. 